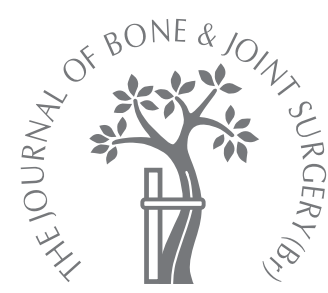

P. T. Hadjicostas, P. N. Soucacos, F. W. Thielemann

From Schwarzwald-

Baar Hospital, Villingen

Schwenningen, Germany

\title{
Computer-assisted osteotomy of the lateral femoral condyle with non-constrained total knee replacement in severe valgus knees
}

\begin{abstract}
We describe the mid-term results of a prospective study of total knee replacement in severe valgus knees using an osteotomy of the lateral femoral condyle and computer navigation. There were 15 knees with a mean valgus deformity of $21^{\circ}\left(17^{\circ}\right.$ to $\left.27^{\circ}\right)$ and a mean follow-up of 28 months (24 to 60). A cemented, non-constrained fixed bearing, posterior-cruciateretaining knee prosthesis of the same design was used in all cases (Columbus-B. Braun; Aesculap, Tuttlingen, Germany).

All the knees were corrected to a mean of $0.5^{\circ}$ of valgus $\left(0^{\circ}\right.$ to $\left.2^{\circ}\right)$. Flexion of the knee had been limited to a mean of $85^{\circ}\left(75^{\circ}\right.$ to $\left.110^{\circ}\right)$ pre-operatively and improved to a mean of $105^{\circ}$ $\left(90^{\circ}\right.$ to $\left.130^{\circ}\right)$ after operation. The mean Knee Society score improved from 37 (30 to 44 ) to 90 points (86 to 94 ).

Osteotomy of the lateral femoral condyle combined with computer-assisted surgery gave an excellent mid-term outcome in patients undergoing total knee replacement in the presence of severe valgus deformity.
\end{abstract}

Joint replacement in a valgus knee requires correction of the deformity and restoration of anatomical alignment to maximise the survival of the implant. This requires correct bony resection, release of lateral soft-tissue structures, ${ }^{1-3}$ tightening of medial soft tissues ${ }^{4}$ and a constrained implant. ${ }^{5,6}$ In valgus deformity, there are primary and secondary abnormalities of bone and soft-tissue. These include contractures of the posterolateral capsule, the iliotibial band and popliteus tendon with lax medial structures. There is also loss of bone in the lateral femoral condyle and central and posterior areas of the lateral tibial plateau. Contracture of lateral soft tissues may prevent correction of the deformity, ${ }^{7}$ and after bony resection soft-tissue stability is difficult to maintain. Although several techniques for balancing of ligaments in the valgus knee have been described, ${ }^{2,8-16}$ balancing of soft tissues and correct alignment of the implant can be difficult.

The use of an image-free navigation system gives the opportunity for a simulation of the planned bony cuts, thereby defining the amount of resection of the distal femoral bone and the selection of the correct femoral implant.

We have attempted to evaluate the outcome of an osteotomy and distal transfer of the lateral femoral condyle in a consecutive series of patients with severe valgus knees due to osteoarthritis. Our technique was combined with computer-assisted surgery (OrthoPilot version 2.0; Aesculap, Tuttlingen, Germany) to manage resection of the femoral bone and to balance the lateral soft tissues.

\section{Patients and Methods}

Between January 2002 and April 2005 we undertook 15 consecutive total knee replacements (TKR) in patients with a pre-operative valgus $>15^{\circ}$ on standing anteroposterior (AP) radiographs. The mean pre-operative femorotibial deformity was $21^{\circ}\left(17^{\circ}\right.$ to $\left.27^{\circ}\right)$. All had a deficient or hypoplastic lateral femoral condyle but none had a large bony defect in the posterior lateral tibial plateau. There were ten women and five men with a mean age of 73 years (64 to 80) and a mean weight of $76 \mathrm{~kg}$ (52 to 98). The diagnosis was osteoarthritis in 13 patients and post-traumatic arthritis in two. Three had had previous arthroscopy and two open medial meniscectomy.

Pre-operatively, a careful physical examination was done to determine the degree of deformity, flexion contracture, range of movement, patellar tracking, ligamentous stability and muscle strength. Radiological assessment included standing AP, lateral, and patellar views, full-length radiographs of the limb and CT of the knee.

Operative technique. A cemented, non-constrained fixed-bearing, posterior cruciate- 
retaining knee prosthesis of the same design (Columbus-B. Braun; Aesculap) was used in all cases.

The operation was performed under general anaesthesia without a tourniquet. The knee was examined to determine whether the valgus deformity was correctable passively and if the medial and lateral aspects of the soft-tissue sleeve were equally taut. A midline skin incision was made. A medial parapatellar arthrotomy was carried out and the medial soft tissues detached subperiosteally from the proximal part of the tibia. Any osteophytes were removed. The patella was everted and dislocated laterally after release of the patellofemoral ligament and the knee was flexed fully to expose the cruciate ligaments and menisci.

The shape of the distal femur was assessed and the knee then extended fully to check for any flexion contracture or recurvatum.

Reference frames were fixed on the distal femur and proximal tibia with bicortical screws, and on the foot with an elastic band. A pivoting algorithm was used to determine the centres of the hip, knee and ankle. Kinematic registration of these joints was used to determine the pre-operative mechanical axes of the limb in the frontal and sagittal planes. The mediolateral laxity in flexion and extension and the range of movement were measured and recorded using the navigation system. A persisting valgus malalignment of $7^{\circ}$ or more under varus stress in full extension suggested that a condylar osteotomy might be required.

Surface landmarks of the tibial plateau and the distal femur were determined by palpation and marked in order to locate the line of the knee joint, the posterior condylar line, the epicondylar line and the anterior cortex. This was necessary to determine the level of femoral and tibial resections in relation to the joint line, to register the epicondylar and posterior condylar lines and the anterior femoral cortex, and to record any deficiency which might involve the lateral femoral condyle, distally and posteriorly, and/or the posterior aspect of the lateral tibial plateau. Distal and posterior bone loss was recorded using a navigated template. All the data were stored on the computer for calculations required later in the operation.

The menisci were excised and the knee flexed maximally and rotated externally to expose the entire tibial plateau. Using the navigation system, the articular surface of the tibia was resected perpendicular to its anatomical mechanical axis. The amount of resection required was usually gauged from the deepest point on the least damaged side of the plateau. The correct orientation of the osteotomy as regards slope, varus-valgus orientation, and level of resection, was recorded by the navigation system.

Laminar spreaders were inserted in the medial and lateral femorotibial spaces with the knee in extension to record the extension gap. They were then used with the knee in $90^{\circ}$ of flexion, thus showing the flexion gap.

With the information from palpation of the landmarks and measurements of the flexion and extension gaps, the final position of the femoral component could be planned.
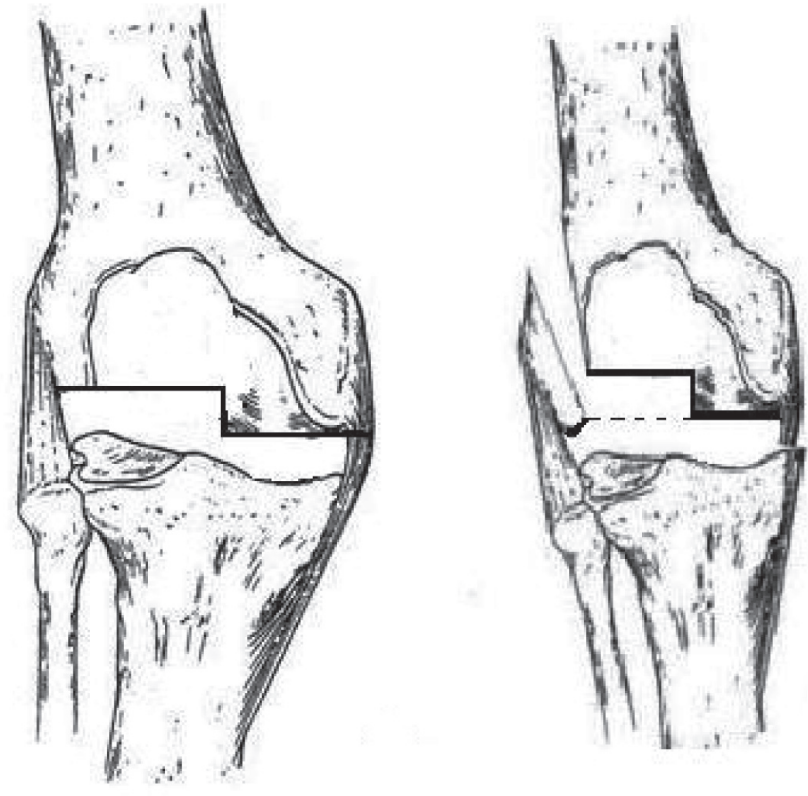

Fig. 1

Drawing showing the step cut

A mathematical model in the software allowed for the selection of the size of the component with the best fit. The level of the distal, anterior and posterior cuts and the rotational position of the component were determined during a simulation with various options. First, rotational alignment of the femoral component was planned to obtain a rectangular space for flexion. Deficiencies of bone stock of the posterior lateral femoral condyle, if present, were ignored at this time. Persistent valgus due to contracted lateral structures was compensated for in the simulation procedure. Flexion and extension gaps were then balanced by selecting the correct size of component in relation to the distal femoral cut. Persistent ligamentous laxity could be corrected by changing the location of the distal cut and size of the femoral or tibial component.

At this stage, deficiencies of bone-stock of the lateral femoral condyle were indicated on the computer screen. A step cut of the lateral femoral condyle was carried out distally (Fig. 1), down to good bone stock, taking into account the difference in height from the planned medial condyle to the planned cut on the lateral condyle, which indicated the distance which the osteotomised lateral condyle had to be transferred distally.

With the information from the simulation and using the computer software under navigation control, the distal cutting block was fixed to the femur. The distal cut perpendicular to the mechanical axis of the femur and the step cut at the lateral condyle were performed and checked. Then, a longitudinal osteotomy of approximately one-third of the width of the lateral condyle was carried out (Fig. 2). The intermuscular septum on the lateral condyle and parts of the 


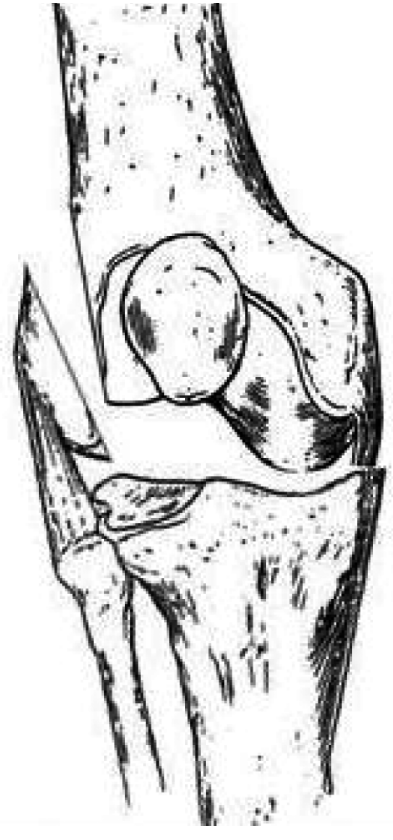

Fig. 2

Drawing showing the condylar osteotomy.

posterolateral capsule were released to allow free movement distally. The lateral condyle was brought level with the medial condyle and temporarily fixed with Kirschner wires.

Afterwards, the correct mediolateral balancing of the extension gap was confirmed by the navigation system. If required, further correction of the position of the lateral condyle was still possible. The condyle was then fixed with two $6.5 \mathrm{~mm}$ cancellous screws with washers. The bony defect on the medial part of the lateral condyle was filled with cancellous bone from the removed tibial plateau (Fig. 3). Then, the rotational position and size of the femoral component were reconfirmed by the simulation software. For correct femoral rotation, the rectangular flexion gap and the Whiteside's line, as described by Amira et al, ${ }^{17}$ had to match. The AP axis was defined as a line connecting the deepest part of the patellar groove anteriorly and the centre of the intercondylar notch posteriorly. The line was approximately perpendicular to the transepicondylar line. Using this reconfirmed information about the planned rotational position of the simulated femoral component, the anterior, posterior and chamfer cuts were made.

When the femoral and tibial resections were completed, a trial reduction was undertaken. The polyethylene insert which optimally balanced the knee in flexion and extension was selected. Special consideration was given to the alignment of the patella, which was usually subluxed in a valgus deformity.

Patellar tracking was evaluated with the trial components in place, using a 'no-thumbs' technique (not pressing the patella with the thumb from lateral to medial to

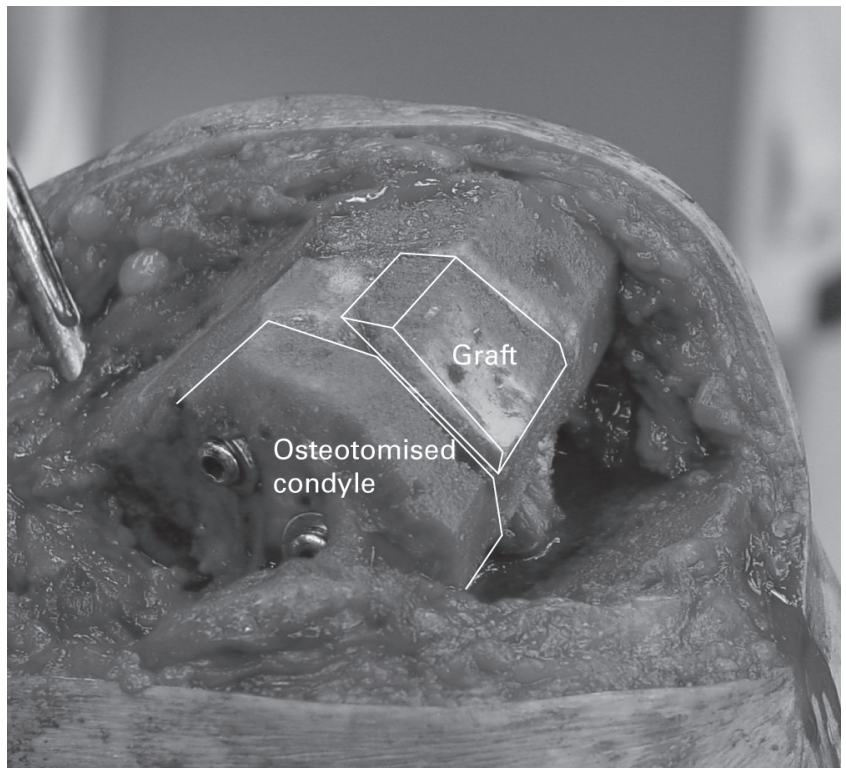

Fig. 3

Photograph showing distalisation of the lateral femoral condyle and fixation with bony filling of the bony step-cut defect.

influence its tracking). The knee was irrigated and the bone dried. Cement was used for fixation of the components and excess cement was removed during pressurisation. Before closure of the wound the final frontal and sagittal alignment and stability under varus and valgus stress in extension and flexion were documented using the navigation system. The range of movement and reconstruction of the Whiteside line were checked. The capsule was closed in flexion over a drain.

A soft dressing was applied to the limb and the patient placed in a continuous passive-motion (CPM) machine in the recovery room. Patients stood with the assistance of a physiotherapist on the second post-operative day, and proceeded to full weight-bearing, using crutches or a walker, on the third post-operative day. All were evaluated closely for signs of injury to the peroneal nerve.

The mean follow-up was 28 months (24 to 60). The patients were evaluated clinically and radiologically at six, 12 and 24 months, and had a post-operative CT. The Knee Society clinical rating system (KSS) was used for pre- and post-operative evaluation. ${ }^{18}$

\section{Results}

The mean pre-operative KSS was 37 points ( 30 to 44 ), which increased to 90 points (86 to 94 ) post-operatively. The corresponding mean functional KSS were 40 (35 to 50) and 90 points (70 to 95 ), respectively. The mean instability score improved from 12 (9 to 13 ) to 21.3 points (15 to 25 ). Pre-operatively, six knees had greater than $10^{\circ}$ varus-valgus laxity, seven between $6^{\circ}$ and $10^{\circ}$ and two less than $6^{\circ}$. 


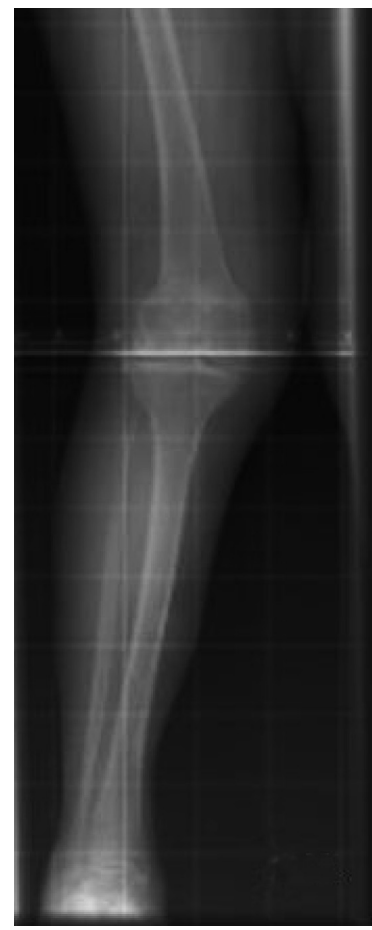

Fig. 4a

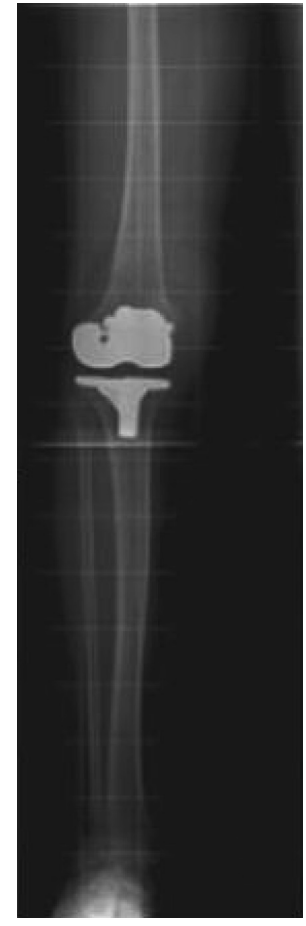

Fig. $4 b$
Long-leg standing radiographs showing valgus deformity of $21^{\circ}$ a) pre-operatively and b) post-operatively after total knee replacement and restoration of normal mechanical alignment ( $1^{\circ}$ of valgus).

Post-operatively, all knees had varus-valgus laxity of less than $6^{\circ}$ in extension, 13 had less than $6^{\circ}$ and two between $6^{\circ}$ and $9^{\circ}$ in flexion.

There was stability in extension and flexion in all patients. They were able to climb stairs without using crutches or a hand rail. Pre-operative flexion was limited to a mean of $85^{\circ}$ $\left(75^{\circ}\right.$ to $\left.110^{\circ}\right)$. The mean flexion post-operatively was $105^{\circ}$ $\left(90^{\circ}\right.$ to $\left.130^{\circ}\right)$. There were no cases of patellar dislocation or palsy of the peroneal nerve. Two patients required lateral release to correct patellar tracking. At follow-up, the patella was recentred in all patients as seen on the post-operative patellar radiograph. All the osteotomies healed and the only complications was an early superficial haematoma which required drainage and irrigation.

The mean pre-operative alignment on AP weight-bearing radiographs was $21^{\circ}$ of valgus $\left(17^{\circ}\right.$ to $\left.25^{\circ}\right)$ which was corrected to a mean of $0.5^{\circ}$ of valgus $\left(0^{\circ}\right.$ to $2^{\circ}$ ) (Figs 4 and 5 ). Pre-operatively, four patients had flexion contractures of between $5^{\circ}$ and $20^{\circ}$. In one with a flexion contracture of $20^{\circ}$, the post-operative flexion contracture was $3^{\circ}$. Preoperatively, transverse CT showed a mean internal rotation of the posterior condylar line of $9.5^{\circ}\left(7^{\circ}\right.$ to $\left.12^{\circ}\right)$. This was corrected post-operatively to $0.5^{\circ}$ of external rotation $\left(2^{\circ}\right.$ internal to $3^{\circ}$ external).

At the time of the last follow-up examination, there was no obvious sign of polyethylene wear or of radiolucencies around the implants.

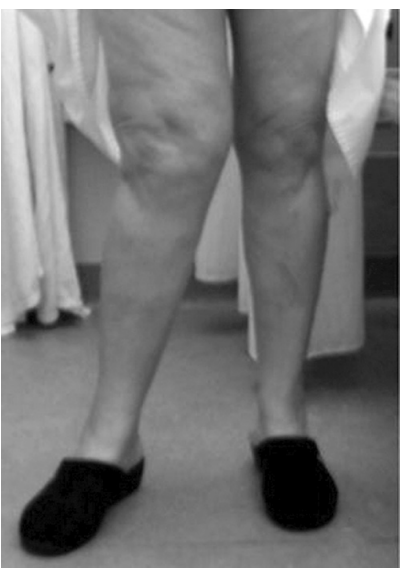

Fig. $5 \mathrm{a}$

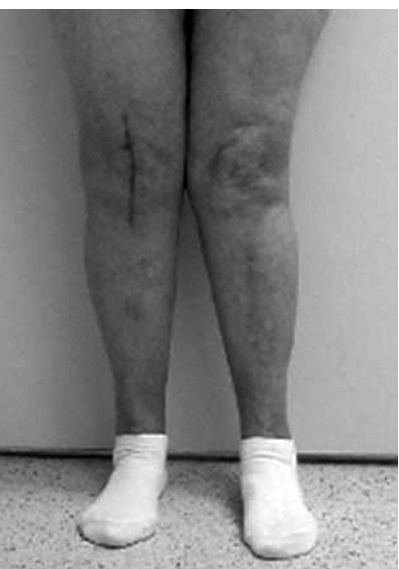

Fig. 5b
Photograph of a 69 -year-old patient showing a) $25^{\circ}$ of valgus pre-operatively and b) $2^{\circ}$ of valgus two years after operation.

\section{Discussion}

The correction of malalignment in valgus knees involves accurate bony resection and soft-tissue balance. Such knees tend to have dysplasia of the lateral femoral condyle and erosion of the lateral tibial plateau, which make it difficult to achieve correct external rotation of the femoral component and may also lead to over-resection of the distal medial femoral condyle.

Whiteside ${ }^{16}$ recommended selective releases of the iliotibial band, popliteus, lateral collateral ligament, and the lateral head of gastrocnemius. He also noted that the popliteus tendon needed to be released only in cases of extreme tightness. If the $\mathrm{Q}$ angle (the angle between anatomical femoral axis and the mechanical axis of the limb) was $>20^{\circ}$, he advised transfer of the tibial tuberosity. Insall and Easley ${ }^{19}$ and Scuderi and Insall ${ }^{20}$ advised the 'pie-crust' technique, which included creation of a symmetrical soft-tissue balance by systematic lengthening of the contracted lateral structures. This was achieved by cutting the arcuate ligament transversely at the level of the tibial cut and progressive lengthening of the iliotibial band and lateral capsule through multiple transverse stab incisions. ${ }^{21}$ Healy et $\mathrm{al}^{12}$ and Krackow et $\mathrm{al}^{4}$ recommended medial soft-tissue advancement combined with lateral soft-tissue releases.

The extended release on the lateral side creates instability in flexion and extension which is reported to be as much as $4 \%$ to $8 \% .^{3,15}$ This can be overcome by the use of a constrained prosthesis or acceptance of some valgus malalignment.

Excessive resection of the medial condyle of the distal femur creates medial ligament laxity in full extension, which can only be corrected by tightening the medial collateral ligament or by using an extremely thick tibial polyethylene insert. If the posterior cruciate ligament (PCL) is intact, the femoral component will roll back excessively and flexion will be compromised. This can be overcome by 
dissection of the PCL with release of the posterolateral capsule, or selection of a smaller femoral component. ${ }^{22,23}$

With the condylar transfer, the extension gap is preserved to a minimal cut while keeping the flexion gap stable. This allows the use of smaller polyethylene inserts and appropriate femoral components. The need for release of the posterior cruciate ligament is minimised and transfer of the femoral condyle with its ligamentous insertions, provides an indirect release of the lateral collateral ligament, popliteus tendon and part of the posterolateral capsule. Bone stock is also preserved for future revision operations. Another important concept is that rotation of the femoral component is determined by balancing of the femoral flexion gap, which has been shown to improve patellofemoral tracking and to produce balanced collateral ligaments. ${ }^{24,25}$

Palsy of the peroneal nerve is cited as a potential problem in knee replacement for valgus deformities with a rate of $3 \%$ to $4 \%{ }^{2,4,15,16,26}$ We had no case of palsy, possibly because our technique did not stretch the nerve at the joint line. Another problem is dislocation, stress fracture or osteonecrosis of the patella. ${ }^{3,4}$ Extensive lateral releases carry a risk of avascularity of the lateral condyle and residual lateral instability. ${ }^{18,19}$ In our group, there were no cases of dislocation, fracture or osteonecrosis of the patella, or residual instability.

Our study had several limitations. First, it lacked a matched group with traditional release of the lateral structures and involved only the use of a non-constrained, posterior cruciateretaining knee prosthesis. Secondly, there were only 15 patients in the series. The use of computer-assisted surgery helped to find the correct position of the lateral epicondyle in order to balance the knee on the lateral side without ligament release. It also provided information for the correct balancing of flexion and extension and rotational alignment. Records of mediolateral laxity in flexion and extension could also be obtained with the navigation system. However, repeated measurements during the operation and the condylar osteotomy and fixation added more time to the procedure. Notwithstanding these disadvantages, we found that, in the medium term this technique was of value in patients presenting with severe valgus deformity.

No benefits in any form have been received or will be received from a commercial party related directly or indirectly to the subject of this article.

\section{References}

1. Whiteside LA. Selective ligament release in total knee arthroplasty of the knee in valgus. Clin Orthop 1999;367:130-40.

2. Stern SH, Moeckel BH, Insall JN. Total knee replacement in valgus knees. Clin Orthop 1991;273:5-8.
3. Favorito PJ, Mihalko WM, Krackow KA. Total knee arthroplasties in valgus knee. $J$ Am Acad Orthop Surg 2002;10:16-24.

4. Krackow KA, Jones MM, Teeny SM, Hungerford DS. Primary total knee arthroplasty in patients with fixed valgus deformity. Clin Orthop 1991;273:9-18.

5. Easley ME, Insall JN, Scuderi GR, Bukkel DD. Primary constrained condylar knee arthroplasty for the arthritic valgus knee. Clin Orthop 2000;380:58-64.

6. Anderson JA, Baldini A, MacDonald JH, Pellici PM, Sculco TP. Primary constrained condylar knee arthroplasty without stem extensions for the valgus knee. Clin Orthop 2006;442:199-203.

7. Scott RD. Total knee arthroplasty. Philadelphia: WB Saunders Co., 2006:45-53.

8. Agglietti P, Buzzi R, Giron F, Zaccherotti G. The Insall-Burstein posterior stabilized total knee replacement in valgus knee. Am J Knee Surg 1996;9:8-12.

9. Aglietti P, Lup D, Cuomo P, Baldini A, de Luca L. Total knee arthroplasty using a piecrusting technique for valgus deformity. Clin Orthop 2007;464:73-7.

10. Buechel FF. A sequential three-step lateral release for correcting fixed valgus knee deformities during total knee arthroplasty. Clin Orthop 1990;260:170-5.

11. Clarke HD, Scuderi GR. Lateral ligament release for valgus deformity in primary total knee replacement. Techniques in Knee Surgery 2003;2:74-81.

12. Healy WL, lorio R, Lemos DW. Medial reconstruction during total knee arthroplasty for severe deformities. Clin Orthop 1998;356:161-9.

13. Krackow KA, Mihalko WM. Flexion-extension joint gap changes after lateral structure release for valgus deformity correction in total knee arthroplasty: a cadaveric study. J Arthroplasty 1999;14:994-1004.

14. Keblish PA. The lateral approach to valgus knee: surgical technique and analysis of 53 cases with over two years follow up evaluation. Clin Orthop 1991;271:52-62.

15. Miyasaka KC, Ranawat CS, Mullaji A. A 10-20 year followup of total knee arthroplasty for valgus deformities. Clin Orthop 1997;345:29-37.

16. Whiteside LA. Correction of ligament and bone defects in total knee arthroplasty for valgus knee. Clin Orthop 1993;288:234-45.

17. Amira J, Whiteside LA, McCarthy DS, White SE. Femoral rotation alignment, based on anteroposterior axis, in total knee arthroplasty in valgus knee: a technical note. J Bone Joint Surg [Am] 1995;77-A:1331-4.

18. Insall JN, Dorr LD, Scott RD, Scott WN. Rationale of the Knee Society clinical rating system. Clin Orthop 1989;248:13-14.

19. Insall JN, Easley ME. Surgical techniques and instrumentation in total knee arthroplasty. In: Insall JN, Scott WN, eds. Surgery of the knee. Third ed. Philadelphia: Churchill Livingstone, 2001:1553-620

20. Scuderi GR, Insall JN. Fixed varus and valgus deformities. In: Lotke PA, ed. Knee arthroplasty. New York: Raven Press, 1995:111-27.

21. Politi J, Scott R. Balancing severe valgus deformity in total knee arthroplasty using a lateral cruciform retinacular release. J Arthroplasty 2004;19:553-7.

22. Matsuda Y, Ishii Y, Noguchi H, Ishii R. Varus-valgus balance and range of movement after total knee arthroplasty. J Bone Joint Surg [Br] 2005;87-B:804-8.

23. Whiteside LA, Summers RG. The effect of the level of distal femoral resection on ligament balance in total knee replacement. In: Dorr LD, ed. The Knee-Papers of the First Scientific Meeting of the Knee Society. Baltimore: University Park Press, 1984:59-73.

24. StiehI JB, Cherveny PM. Femoral rotational alignment using tibial shaft axis in total knee arthroplasty. Clin Orthop 1996;331:47-55.

25. Olcott CW, Scott RD. Femoral component rotation during total knee arthroplasty. Clin Orthop 1999;367:39-42.

26. Clarke HD, Schwartz JB, Math KR, Scuderi GR. Anatomic risk of peroneal nerve injury with the 'pie crust' techniquer for valgus release in total knee arthroplasty. $J$ Arthroplasty 2004;19:40-4 